\title{
Paracoccidioidomicosis: Manifestaciones orales e implicaciones sistémicas
}

\section{Paracoccidioidomicose: Oral manifestations and sistemic implications}

\author{
Janete Grando L*, Somacarrera Pérez ML**, Luckmann Fabro SM***, \\ Meurer MI***, Riet Correa Rivero E****, Modolo F****
}

\section{RESUMEN}

La paracoccidioidomicosis es una infección causada por un hongo $P$. brasiliensis. Se trata de una micosis profunda y sistémica, es considerada una enfermedad endémica en Brasil y en otros países de América Latina. El hongo es adquirido por inhalación, dando lugar a un cuadro clínico pulmonar que puede posteriormente afectar a la piel, mucosa oral, nasal y gastrointestinal; también el bazo y el hígado pueden verse afectados. Es frecuente encontrar linfadenopatías, y verse afectada la glándula suprarrenal y todas las vísceras, causando la muerte del paciente. Las úlceras orales suelen ser lesiones muy dolorosas, por ello el paciente acude a consulta para ser evaluado clínicamente por su odontólogo o estomatólogo. El diagnóstico precoz es la mejor manera de salvar al paciente de las complicaciones de la enfermedad. El número creciente de inmigrantes latinos puede hacer que esta importante enfermedad empiece a ser más prevalente en los países de la Comunidad Europea, por ello conocer en profundidad las características clínicas de esta infección por parte de los odontólogos europeos, es fundamental para llegar a diagnosticarla de forma temprana.

Palabras clave: Paracoccidioidomicosis, blastomicosis sudamericana, blastomicosis brazilensis.

\section{SUMMARY}

Paracoccidioidomycosis is an infection caused by the $p$. brasiliensis fungus. This is a deep, systemic fungal infection considered endemic in Brazil and some Latin American countries. The fungus is acquired by inhalation, resulting in a pulmonary disease which may also affect the skin, oral, nasal and gastrointestinal mucosa, as well as the spleen and liver. Patients commonly present lymphadenopathy. The resulting adrenal gland and visceral involvement can be fatal.

Oral lesions are often very painful leading the patient to the dentist or stomatologist for a clinical evaluation. Early diagnosis is the best way to avoid serious complications of the disease.

The growing number of Latin immigrants may increase the prevalence of this important disease in the European Community, therefore, the knowledge of the clinical characteristics of this infection by European dentists is essential to early diagnosis.

Key words: Paracoccidioidomycosis, blastomycosis sulamerican, blastomycosis brazilensis.

* Profesora. Ambulatório de Estomatologia. Hospital Universitário. Universidade Federal de Santa Catarina (UFSC), Brasil. Becaria de la Fundación Carolina, España. Beca de Movilidad de Profesores Brasileños.

** Catedrática de Medicina Oral. Departamento de Odontologia. Facultad de Ciencias de La Salud. Universidad Europea de Madrid.

*** Profesora. Ambulatorio de Estomatologia. Hospital Universitário. UFSC, Brasil.

**** Profesor/a. Laboratorio de Patología Bucal. Curso de Odontología/UFSC, Brasil. 
Fecha de recepción: 10 de diciembre de 2009.

Aceptado para publicación: 21de diciembre de 2009.

Janete Grando L, Somacarrera Pérez ML, Luckmann Fabro SM, Meurer MI, Riet Correa Rivero E, Modolo F. Paracoccidioidomicosis: Manifestaciones orales e implicaciones sistémicas. Av. Odontoestomatol 2010; 26 (6): 287-293.

\section{INTRODUCCIÓN}

La enfermedad Paracoccidioidomicosis (PCC) es una infección profunda, sistémica, que tiene como agente causal el hongo Paracoccidioides brasiliensis, cuyo hábitat natural es la tierra $(1,2)$.

La paracoccidioidomicosis o blastomicosis suramericana es una enfermedad micótica sistémica progresiva, común en Latinoamérica. Esta infección fúngica se encuentra en focos endémicos en la zona que se extiende entre México por el norte hasta Argentina por el sur, con la mayoría de los casos en Brasil, Venezuela y Colombia. Fue descrita por primera vez por Adolfo Lutz, en 1908. Después de 4 años, Splendore describió la morfología del hongo y detalló cuatro casos en la Santa Casa de Misericordia de São Paulo. En 1930, Floriano Paulo de Almeida denominó a este hongo como Paracoccidioides brasiliensis. Por esto, la infección fue inicialmente llamada "molestia de Lutz-Splendore-Almeida". En 1971, el nombre Paracoccidioidomicosis fue instituido, persistiendo hasta la actualidad (1).

Este hongo asexuado es termodimórfico, es decir, presenta dos formas: la primera es la de micelio, forma infectante, a la temperatura ambiente, 23 a $28^{\circ} \mathrm{C}$; en esta forma, se observa la presencia de hifas delgadas, enredadas y elongadas: La segunda forma es de levadura, forma diseminante, a temperatura de 35 hasta los $37^{\circ} \mathrm{C}$ y se caracteriza por la presencia de células esféricas u ovaladas, con paredes bien definidas, birrefringentes, duplicadas, que pueden presentar brotes multipolares característicos, las formas clásicas denominadas de "orejas de Mickey Mouse" o "rueda de timón (3).

La Paracoccidioidomicosis (PCC) es una infección autóctona, es decir, natural del país donde vive y proveniente de las etnias que siempre han vivido en el (2)
En Brasil es considerada una enfermedad endémica, observada en los estados del Río Grande do Sul, Paraná, São Paulo, Río de Janeiro, Minas Gerais, Mato Grosso do Sul y regiones del cerrado de Goiás y Mato Grosso (2)

Para SANTO (4), la PCC es la infección micótica sistémica más importante en Brasil, donde se puede encontrar el mayor número de zonas endémicas de todo el mundo. Por la gran cantidad de muertes precoces que provoca en los trabajadores del campo, la PCC es considerada un grave problema de salud pública en Brasil y en los países de América del Sur. Los hombres se afectan con mayor prevalencia que las mujeres, en edades que oscilan de los 55 y los 64 años, en su gran mayoría son trabajadores de áreas rurales, agricultores y trabajadores de la construcción.

La entrada del hongo al individuo ocurre tras su inhalación, con la llegada de este a los pulmones. El período de incubación en los pulmones puede ser de un mes hasta muchos años. No hay riesgo de transmisión de una persona a otra. La contaminación tras heridas en la piel o mucosas aunque ha sido descrita es extremadamente rara (3).

En los adultos, la forma clínica predominante es la crónica, puede afectar a niños y adolescentes, de manera aguda o subaguda ("PCC juvenil"), comprometiendo en especial los ganglios linfáticos, bazo e hígado, dando lugar a disfunción de la medula ósea (4).

En las mujeres, el hongo en su forma miscelánea, sufre la acción de la hormona femenina 17-B-estradiol, volviéndose incapaz de transformarse de la forma micelar (forma infectante) a la forma de levadura (diseminante), fundamental para la producción de la enfermedad PCC (3).

En los pacientes adultos, la afectación más grave es la de los pulmones. Puede presentar regresión es- 
pontánea, pero es rara. Puede afectar la piel, mucosa oral, nasal y gastrointestinal, el bazo, el hígado y acompañarse de linfadenopatías. En su evolución clínica, se puede producir empeoramiento y puede afectar la glándula suprarrenal y todas las vísceras, causando la muerte del paciente (4).

Las manifestaciones clínicas y la afectación sistémica son muy importantes y suelen tener un tiempo de duración muy largo. Un paciente que se ha infectado en la adolescencia puede presentar las manifestaciones clínicas después de muchos meses o incluso cuando es adulto. El adecuado diagnostico y vigilancia incluye la realización de exámenes por imagen, radiografías de tórax y abdomen, analítica de sangre (hemograma completo, velocidad de sedimentación), analítica hepática (ALT, AST, gGT, fosfatasa alcalina), electroforesis de proteínas, analítica renal y metabólica (creatinina, niveles de sodio y potasio) (SHIKANAI-YASUDA et al, 2005)

Los test hematológicos pueden revelar la presencia del hongo antes de los demás test de cultivo o histopatológico. Se pueden detectar los anticuerpos IgM e IgA del $P$. brasiliensis en el suero del paciente $(1,5)$ se utilizó de la técnica de inh-ELISA para detección de la glicoproteína $43 \mathrm{Kda}$, un antígeno exocelular secretado durante la fase de infección por el hongo, en fluido cerebroespinal y lavado bronco alveolar, con un grado de fiabilidad de $100 \%$.

La PCC afecta más frecuentemente a los pacientes con patología sistémica asociada, infección por el $\mathrm{VIH}$, hepatitis, tuberculosis, enfermedad pulmonar obstructiva crónica, linfoma de Hodgkin, carcinomas y otras situaciones como la desnutrición severa, alcoholismo, tabaquismo, o la utilización de drogas. Si el proceso no es diagnosticado y tratado oportunamente, la PCC puede ser letal (3).

El conocimiento de esta enfermedad por los dentistas y estomatólogos de los países de la Comunidad Europea es importante, debido al aumento en el número de inmigrantes sudamericanos.

\section{CARACTERÍSTICAS CLÍNICAS Y DIAGNÓSTICO DE LA PCC}

El principal motivo de una cita de pacientes con PCC es la perdida significativa de peso en corto periodo de tiempo, astenia, y sensación de cansancio para las actividades cotidianas. Por esto, el médico suele ser el primer profesional consultado.

Desde el punto de vista radiológico probablemente las lesiones iniciales tienen apariencia de vidrio esmerilado con algunas lesiones acinares y algún espesamiento del intersticio axial probablemente debida a diseminación linfática. Hay que destacar la reducción del volumen pulmonar, lesiones bilaterales, simétricas y de carácter retráctil, padrones lineales y nodulares heterogéneos de los dos pulmones. A medida que la enfermedad se disemina se pueden hallar toda clase de lesiones con la destrucción del árbol pulmonar tendremos hiperinsuflación, bullas subpleurales y/o espesamiento del intersticio axial pudiendo llegar a mostrar panalización $(6,7)$.

Las manifestaciones bucales más importantes son la macroqueilia (Fig. 1A) y las úlceras dolorosas y esto puede ser el motivo de una primera consulta al odontólogo o estomatólogo. Las úlceras son rasas, generalmente múltiples, con sus contornos y bordes irregulares, fondo granuloso, de coloración amarilla y con puntos rojos (clásico aspecto de piel de fresa "estomatitis moriforme de Aguiar-Pupo"), que afectan más la mucosa labial (Figs. 1B y 1E), mucosa alveolar (Fig. 1D), las encías y el paladar Fig. 1C). Las úlceras producen dolor espontáneo o provocado por la masticación, dificultando la alimentación y la higiene oral, y desarrollando una afectación importante de la salud del paciente.

Durante el proceso de cicatrización de las úlceras, puede producirse una fibrosis muy intensa y variable de los tejidos blandos, con una importante reducción del perímetro bucal, microstomía. Las manifestaciones en la boca son las que más molestias causan a los pacientes y su diagnóstico rápido es fundamental (2).

Los estudios de microscopia mediante citología exfoliativa fueron indicados como un método auxiliar útil al diagnóstico clínico, de fácil realización, de bajo coste, que no causa daño a los pacientes. Cuando se asocia con la técnica de impregnación por plata (GOMORI-GROCOTT) es posible una mejor identificación del hongo $P$. brasiliensis. La 

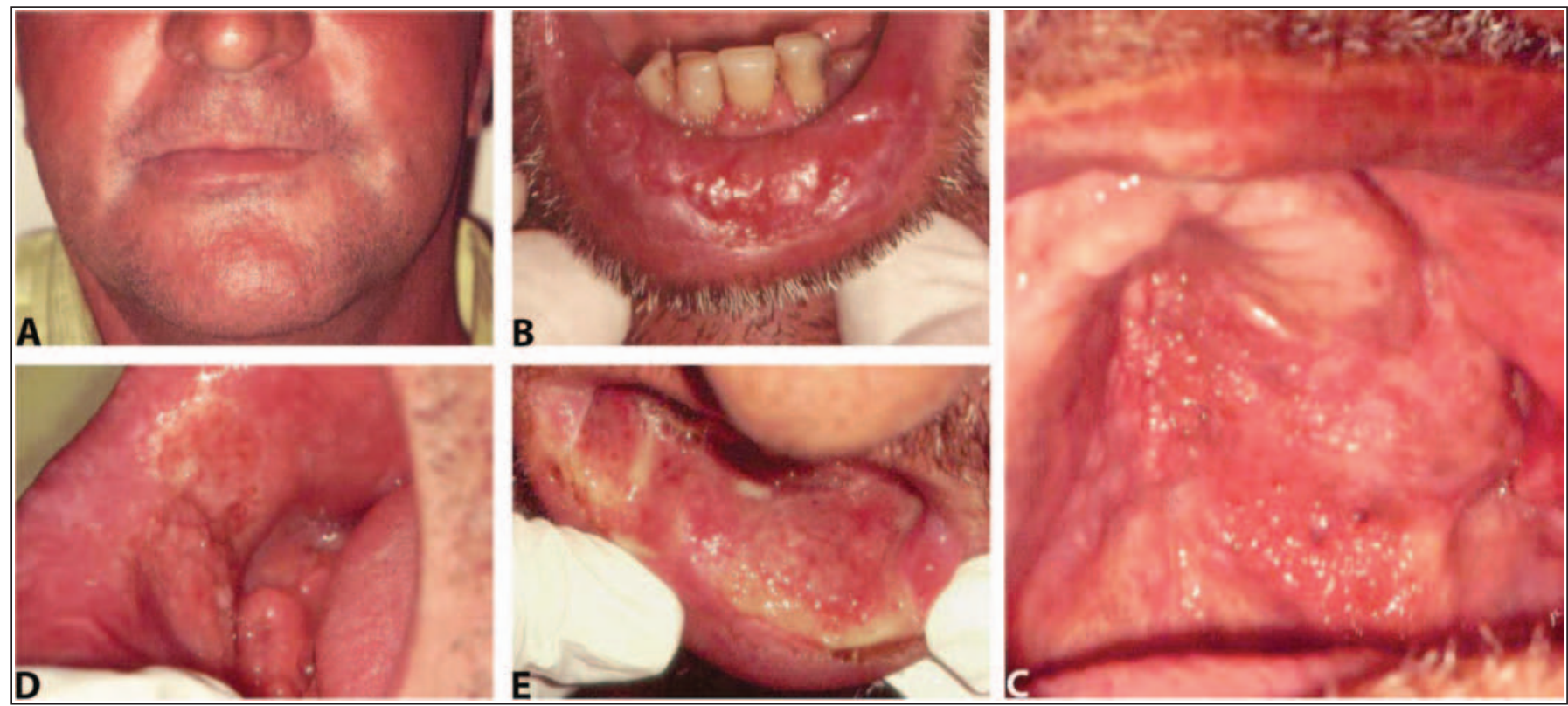

Fig. 1. Aspectos clínicos de PCC en diferentes pacientes. A: Macroqueilia en hemilábio inferior derecho. B: Úlceras en mucosa y semi-mucosa labial inferior. C: úlceras en paladar duro y blando. D: Úlceras de aspecto moriforme en mucosa labial inferior y reborde alveolar derecho. E: Úlceras en mucosa labial inferior (Imágenes de los autores).

histopatología de los tejidos biopsiados (biopsias incisionales) deben ser procesadas con tinción de HEE y de GOMORI-GROCOTT (8).

En los cortes histopatológicos de una úlcera bucal, se observa la presencia de un epitelio plano estratificado, con espongiosis e hiperplasia pseudoepiteliomatosa (Fig. 2A.1). En el corion subyacente, se observa una inflamación granulomatosa difusa, con muchas células gigantes multinucleadas (Figs. 2A.2 y 2B) con el hongo $P$. brasiliensis en su interior (Fig. 2C) y un intenso infiltrado inflamatorio linfoplasmocitario. En el examen de citología las levaduras se identifican mejor con la impregnación de plata (tinción de Grocott-Gomori). Los hongos muestran brotes hijos, conectados a sus células madre, proceso de gemación, dando lugar a un aspecto clásico, ya bien descrito como de "rueda de timón" (Fig. 2D) o de "orejas de Mickey Mouse" (Fig. 2E).

\section{PRONÓSTICO}

El pronóstico de la PCC es sombrío, pues es muy difícil que el paciente cure por completo, necesitan-

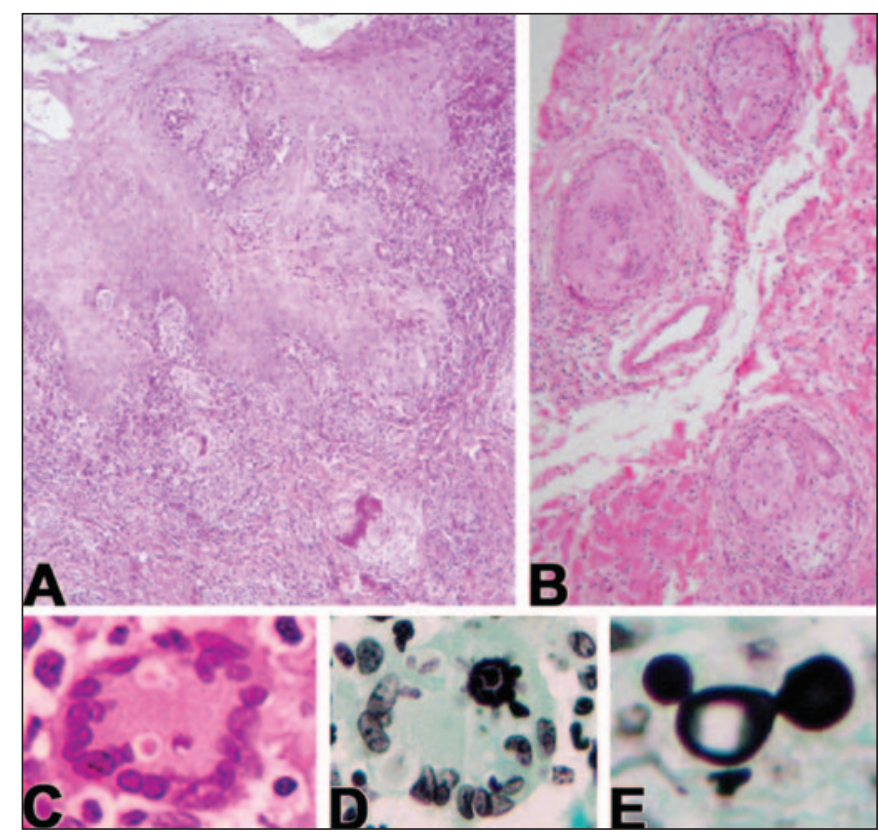

Fig. 2. Aspectos histopatológicos de la PCC. A: Epitélio con hiperplasia pseudoepiteliomatosa (A.1) y tejido conjuntivo con muchas células gigantes multinucleadas (A.2). B: Formando granulomas. C: Presencia de $p$. Brasiliensis (flechas) dentro de una célula gigante multinucleada. D, E: Citología exfoliativa, con hongo haciendo esporulación múltiple. D: Se puede observar un brote del hongo en "rueda de timón". E: Hongo en "orejas de mickey mouse" (tinción de Gomori-Grocott) (Imágenes de los autores). 
do generalmente ser revisado de forma periódica. Las opciones terapéuticas son variables y dependen de las condiciones del sistema de salud pública del país, de la severidad de las manifestaciones clínicas y del estado inmunológico del paciente (3).

\section{TRATAMIENTO}

El tratamiento de las micosis profundas y/o sistémicas se ha venido haciendo desde 1978 con Anfotericina $\mathrm{B}$ y/o Flucitosina. Los imidazoles: miconazol y ketoconazol, fueron introducidos durante los siguientes tres años. El tratamiento consiste en dos fases: inducción y mantenimiento. El tratamiento de ataque tiene como objetivo hacer un inmediato control de los signos y síntomas de la PCC, reduciendo la carga de hongos circulantes. El tratamiento de mantenimiento es siempre de larga duración, desde muchos meses hasta años $(9,10)$.

A continuación presentamos algunas de las pautas terapéuticas utilizadas:

a) Trimetropin-Sulfametoxazol: 800/60 mg/día, vía oral, cada 12 horas, durante 30 días, seguido de 400/80 mg cada 12 horas, hasta un año después de que la serología se haga negativa.

b) Ketoconazol: $400 \mathrm{mg} /$ día, vía oral, durante 45 días, seguido de 200 mg/día hasta completar 12 meses. El ketoconazol administrado vía oral es una opción efectiva, no costosa y conveniente para tratar candidiasis mucosa, y fue ampliamente utilizada durante toda una década ya que no existía ningún otro agente antimicótico efectivo para tratar micosis sistémicas profundas. Durante la década de los 90‘ el uso del ketoconazol disminuyó debido a la aparición de los triazoles: fluconazol e imidazol.

c) Fluconazol: $400 \mathrm{mg} /$ día, vía oral, durante un mes, seguido de $200 \mathrm{mg} /$ día, durante 6 meses. Este fármaco es la mejor opción para el tratamiento da neuroparacoccidioidomicosis, debido a su elevada concentración en el SNC; la dosis de ataque puede llegar hasta $800 \mathrm{mg}$ /día durante 30 días El fluconazol es menos tóxico y tiene algunas ventajas farmacológicas sobre el ketoconazol, como la penetración dentro del líquido cerebroespinal; además tiene una eficacia mayor en contra de candidiasis, criptococosis y coccidioidomicosis sistémicas.

d) El Itraconazol de 100 a 400 mg/día. Es un antimicótico derivado de los triazoles de espectro muy amplio. Cuando se administra oralmente, el Itraconazol alcanza altos niveles de concentración y los mantiene en muchos tejidos, como la piel, las uñas y la mayoría de los órganos profundos. Su valor clínico ha sido demostrado en todas las formas de micosis que afectan piel y mucosas y en las onicomicosis. Es la droga de elección para infecciones sistémicas como blastomicosis, histoplasmosis, paracoccidioidomicosis, esporotricosis linfocutánea, aspergilosis crónica diseminada y muchos casos de candidiasis y criptococosis profundas y de largo tiempo de evolución, aún cuando la infección micótica ya haya alcanzado las meninges $u$ otro órgano del SNC.

e) Anfotericina B: $1 \mathrm{mg} / \mathrm{kg} /$ día, IV, diluido en $50 \mathrm{ml}$ de suero glucosado al $5 \%$ más acetato de delta hidrocortisona 50-100 mg. La dosis máxima de anfotericina B no debe de ser superior a $3 \mathrm{~g} /$ día. Para uso hospitalario. La anfotericina sigue siendo la terapia estándar de elección para las micosis más profundas, mientras que los nuevos azoles son los agentes de primera línea para las micosis superficiales como la candidiasis, y su uso está actualmente incrementándose en las micosis profundas.

\section{PRESENTACIÓN DE UIN CASO CLÍNICO}

Varón de 45 años de edad, agricultor, fumador desde hace más de 20 años, fue remitido debido a una dificultad de cicatrización en un área sometida a exodoncia previa en región anterior inferior. Durante el examen físico, el paciente relató importante pérdida de peso en los últimos 6 meses, más de 10 kilos (Fig. 3A). A la exploración se observa macroqueilia y la presencia de varias úlceras crateriformes, rasas, dolorosas, con fondo granulomatoso, base indurada y con puntos eritematosos en las regiones de reborde alveolar anteroinferior y en mucosa del labio inferior (Fig. 3B) y de trígono retromolar izquierdo que se extienden hasta paladar blando y mucosa yugal izquierda (Fig. 3E).

Bajo la sospecha clínica de PCC, fueron solicitadas radiografías de tórax posteroanterior y lateral y una 


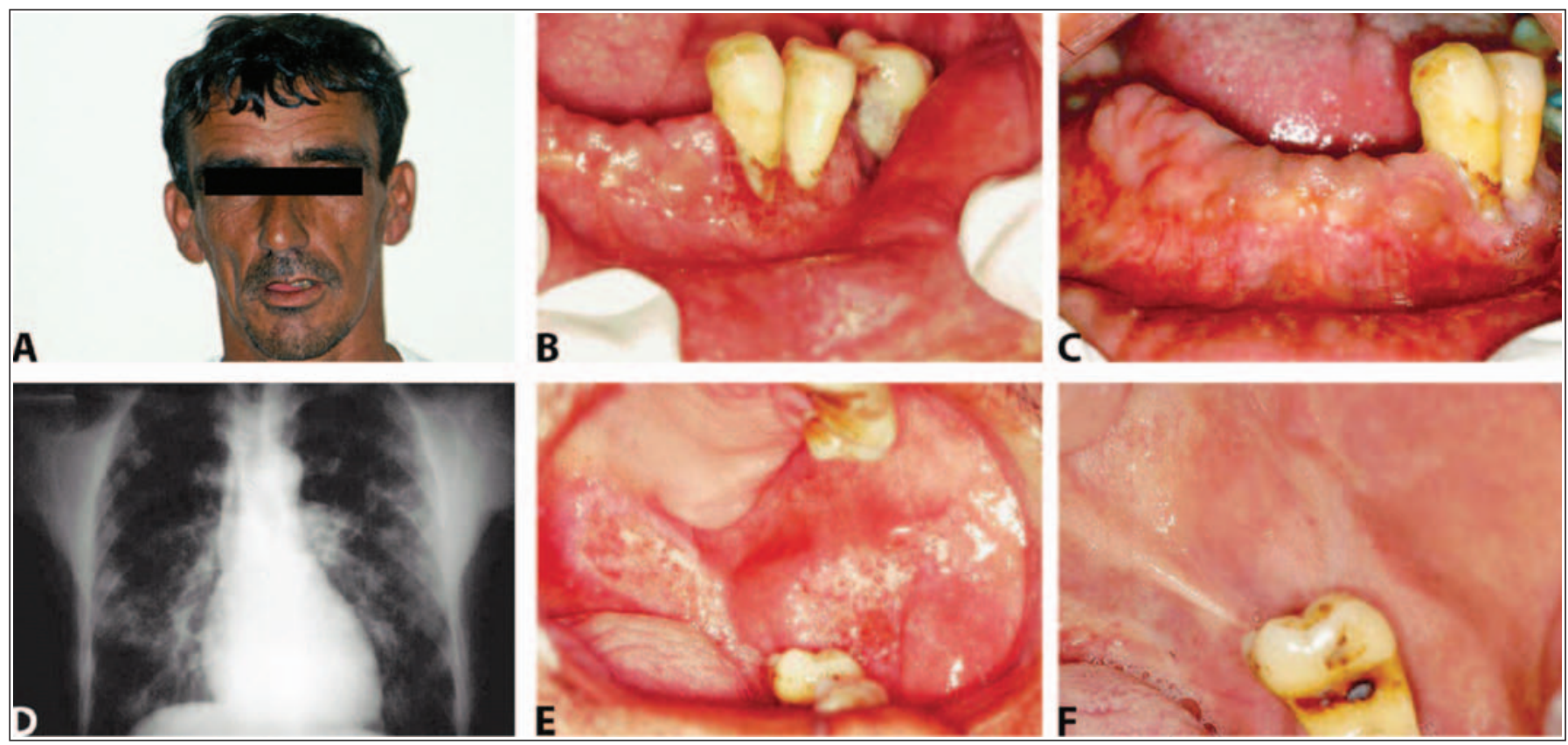

Fig. 3. A: la cara del paciente, con perdida de peso y macroqueilia inferior. B: Úlceras en mucosa alveolar y labio inferior antes del tratamiento. C: Mejoría de los tejidos después del tratamiento. D: Los dos pulmones afectados por PCC. E: Dos ulceras en trígono retromolar y mucosa yugal izquierda antes del tratamiento. F: Normalidad de los tejidos después del tratamiento (Imágenes de los autores).

analítica completa. Se realizó biopsia incisional de la ulcera del labio inferior; el examen histopatológico fue compatible con el diagnóstico clínico. Las radiografías de tórax revelaron afectación pulmonar (Fig. 3D) y la analítica mostraba afectación de las glándulas suprarrenales (enfermedad de Addison).

El tratamiento de inicio fue Ketoconazol 200 mg, dos veces al día y Prednisona $5 \mathrm{mg}$ al día. El paciente ha respondido al tratamiento de inducción que se mantuvo cuarenta y cinco días y fue posteriormente seguido con mantenimiento a base de Ketoconazol 200 mg/día y reducción gradual de la Prednisona, hasta los 18 meses posteriores. Hubo una mejoría significativa de las lesiones bucales (Figs. 3C y 3F), el paciente recuperó tres kilos de peso en 30 días. El paciente se encuentra bajo riguroso control y vigilancia clínica por los equipos de Medicina Interna y de Estomatología.

\section{CONCLUSIONES}

La paracoccidioidomicosis es una infección producida por el hongo $P$. brasiliensis, se trata de una micosis profunda, muy grave, que puede comprometer múltiples órganos internos y causar la muerte del paciente.

Las manifestaciones en boca especialmente la macroqueilia y las úlceras mucosas dolorosas pueden llevar al diagnóstico clínico de esta enfermedad por parte del odontólogo o estomatólogo. El diagnóstico precoz es la mejor manera de salvar al paciente de las complicaciones de la enfermedad.

El número creciente de inmigrantes latinos puede hacer que esta importante enfermedad empiece a ser cada vez más diagnosticada en los países de la Comunidad Europea.

\section{BIBLIOGRAFÍA}

1. Palmeiro M, Cherubini K, Yurgel LS. Paracoccidioidomicose - Revisão da Literatura. Scientia Medica PUCRS 2005;15(4):274-8.

2. Verli FD, Marinho AS, Souza SC, Figueiredo MA, Yurgel LS. Perfil clínico-epidemiológico dos pa- 
cientes portadores de paracoccidioidomicose no Serviço de Estomatologia do Hospital São Lucas da Pontifícia Universidade Católica do Rio Grande do Sul, Rev Soc Bras Med Tropical 2005;38(3):234-7.

3. Shikanai-Yasuda MA, Telles Filho FQ; Mendes RP; Colombo AL, Morettis M. Consenso em paracoccidioidomicose. Rev Soc Bras Med Tropical 2006;39(3):297-310.

4. Santo AH. Paracoccidioidomycosis-related mortality trend, state of São Paulo, Brazil: a study using multiple causes of death. Rev Panam Salud Publica 2008;23(5):313-24.

5. Marques da Silva SH, Colombo AL, Blotta MHSL, et al. Detection of circulating gp43 antigen in serum, cerebrospinal fluid and bronchoalveolar lavage fluid of patients with paracoccidioidomycosis. J Clin Microbiol 2003;41:3675-80.

6. Pérez D, Oviedo J, Gill S. Paracoccidioidomicosis: características clínicas y evolutivas de 94 casos. Instituto Nacional de Enfermedades Respiratorias y del Ambiente (INERAM). VII Congreso Paraguayo de Medicina Interna: Asunción, Paraguay 2004.
7. Recalde V, Oviedo J, Gill S, Pérez D. Paracoccidioidomicosis: descripción de 113 casos (Programa de Residencia en Neumología). Instituto Nacional de Enfermedades Respiratorias y del Ambiente (INERAM). Asunción, Paraguay 2005: 17 p.

8. Araújo M, Sousa SCOM, Correia D. Avaliação do exame citopatológico como método para diagnosticar a paracoccidioidomicose crônica oral. Rev Soc Bras Med Tropical 2003;36(3):427-30.

9. Negroni, R. Evolución de los conocimientos sobre aspectos clínico-epidemiológicos de la coccidioidomycosis en las Américas. Rev Argent Microbiol 2008; 40(4):246-56.

10. Casbarro Arriaga R, Jiménez CC, Tinoco PJ, Machado E. Paracoccidioidomicosis sistémica con implicación de la cavidad bucal. Acta Odontológica Venezolana, 2001;39(2).

\section{CORRESPONDENCIA}

María Luisa Somacarrera Pérez

Avenida de América, 37

28002 Madrid

Correo electrónico: mluisa.somacarrera@uem.es 